\section{NONPARAM: A BASIC program package for nonparametric procedures}

\author{
GERNOT von COLLANI \\ Technical University of Brunswick \\ Braunschweig D-3300, West Germany
}

The program described here is written in Level II BASIC for the TRS-80, but it may easily be adapted to other machines with Microsoft BASIC.

Nonparametric Methods. The most common nonparametric test procedures described by Hays (1963), Kirk (1968), Siegel (1956), and Winer (1961) are included in the program package described here, except for goodness-of-fit tests. The tests are the Kruskal-Wallis one-way analysis of variance ( $\mathrm{H}$ test), the Friedman twoway analysis of variance, the Mann-Whitney $U$ test, the Wilcoxon test for matched pairs, the McNemar test for correlated proportions, Cochran's Q, Fisher's exact probability test, the binomial test, the Wald-Wolfowitz runs test, and, as correlation methods, Spearman's rho, Kendall's W, Kendall's tau, and Goodman and Kruskal's gamma.

Computational Procedures. The test statistic for the Friedman test corrected for ties is computed here by means of an analysis of variance method proposed by Winer (1961, p. 136f). It may be shown that, with ties, this method of computation yields a test statistic that is algebraically related to Kendall's coefficient of concordance (W) corrected for ties. If ties are present in both variables, Kendall's tau is computed following the procedure described by Hays $(1963, p .652 f)$. The case of zero differences in Wilcoxon's matched-pairs test is handled in the following way: If there are an even number of zero differences, an average rank is allocated to the whole set and half of the differences are arbitrarily given a positive and the rest a negative sign. In the case of an odd number of zero differences, one randomly selected difference is canceled and the rest are treated as in the case of even differences.

Sorting Procedure. For some nonparametric procedures, the original data must be converted to ranks; that is, they must be sorted according to numerical value before they are assigned their proper ranks. The sorting procedure used here is a variety of QUICKSORT (Hoare, 1962).

Program Organization. The selection of a particular test procedure is accomplished via a menu. The only input device provided is the keyboard. The user is guided toward entering the necessary control data. All other data input requires the original data, that is, the data that are not yet transformed into ranks, if applicable. The output on the display consists of the test statistics with and without correction for ties, as well as some additional information that might be valuable to the user (e.g., rank sums, $\mathrm{z}$ approximation, if applicable). The output is organized in such a way that it occupies one page on the screen. Users desiring a hard copy may route the output from the screen to a printer or may utilize the hard-copy facilities offered by several disk operating systems. The TRS- 80 Model 3 even has a built-in hardcopy feature.

Requirements. The program package was written in BASIC Level II on a TRS-80 Model 1 with $48 \mathrm{~KB}$ and two floppy disk drives. The program text, including comments, occupies approximately $18 \mathrm{~KB}$ of RAM. The program will also run on TRS.80 Models 1 and 3 equipped with a tape recorder and at least $32 \mathrm{~KB}$ of RAM. No features of disk BASIC are used.

Availability. A program listing is available free of charge from the author on request. If a 5.25 -in. diskette is provided and International Reply Coupons worth $\$ 3$ for postage are added, a copy of the program will be supplied on the disk.

\section{REFERENCES}

HAys, W. L. Statistics for psychologists. New York: Holt, Rinehart \& Winston, 1963.

HoAre, C. A. R. QUICKSORT. Computer Journal, 1962, 5, 10-15.

KIRK, R. E. Experimental design. Belmont: Wadsworth, 1968. Siegel, S. Nomparametric statistics. New York: McGraw-Hill, 1956.

WiNER, B. J. Statistical principles in experimental design. New York: McGraw-Hill, 1961.

(Accepted for publication November 17, 1982.) 